\title{
Wirksame Aufklärung durch Medizinstudentin im Praktischen Jahr
}

\section{Zusammenfassung}

Die Eingriffs- und Risikoaufklärung ist eine der wesentlichen Pflichten des Arztes im Behandlungsverhältnis. Eine Delegation der Aufklärung an andere Ärzte ist daher nur unter bestimmten Voraussetzungen zulässig, eine Übertragung auf nichtärztliches Personal grundsätzlich ausgeschlossen. Dass dies nicht ausnahmslos gilt, zeigt ein aktuelles Urteil des Oberlandesgerichts (OLG) Karlsruhe vom 29.01.2014 - 7 U 163/12 - . Das Gericht bewertete die Aufklärung durch eine Medizinstudentin im Praktischen Jahr (PJ) als zulässig. Nachfolgend sollen die Voraussetzungen dargestellt werden, unter denen eine Aufklärung in Ausnahmefällen wirksam auch von Studenten im Praktischen Jahr übernommen werden kann.

\section{Text}

\section{Zum Sachverhalt}

Eine Patientin hatte auf Zahlung von Schmerzensgeld geklagt, nachdem sie sich in der beklagten Klinik einer Herzkatheteruntersuchung unterzogen hatte. Dabei war es zu einer Gefäßverletzung in der Leistengegend gekommen, die erst nach einigen Tagen diagnostiziert und behandelt worden war. Ihre Forderung begründete die Patientin in erster Linie mit dem Vorwurf der fehlerhaften bzw. unzureichenden Aufklärung: Die Eingriffs- und Risikoaufklärung hatte vor der Operation nicht der behandelnde Arzt, sondern eine Medizinstudentin vorgenommen, die gerade ihr Praktisches Jahr in der Klinik absolvierte. Ob bei diesem Aufklärungsgespräch auch ein Arzt zugegen war, ließ sich nachträglich nicht mehr feststellen, es wurde aber von der Studentin explizit auf Gefäßverletzungen hingewiesen. Nach Auffassung der Patientin war die Aufklärung durch die Medizinstudentin unzulässig und damit ihre eigene Einwilligung in den Eingriff unwirksam und der Eingriff rechtwidrig. Ebenso wie das erstinstanzlich angerufene Landgericht Karlsruhe kam jedoch auch das OLG zu dem Schluss, dass die Übertragung der Aufklärung auf einen Medizinstudenten im praktischen Jahr bei Vorliegen besonderer Voraussetzungen im Einzelfall zulässig sein kann und - bei inhaltlicher Richtigkeit und Vollständigkeit - kein Aufklärungsfehler allein durch diesen Umstand begründet werden kann.

\section{Die Entscheidung}

Das Gericht bewertete die durch die Studentin erfolgte Eingriffs- und Risikoaufklärung weder inhaltlich als unzureichend noch deshalb als unbeachtlich, weil sie von einer Medizinstudentin im Praktischen Jahr durchgeführt worden war. Das OLG berief sich insoweit auf die Rechtsprechung des Bundesgerichtshofs (BGH), wonach der Patient „im Großen und Ganzen“ wissen müsse, worin er einwil- lige. Dazu müsse der Patient über die Art des Eingriffs und seine nicht ganz außerhalb der Wahrscheinlichkeit liegenden Risiken informiert werden. Jedenfalls inhaltlich war demnach die Aufklärung durch die Medizinstudentin nicht zu beanstanden: So hatte sie die Patientin insbesondere darauf hingewiesen, dass es dort, wo der Katheter entlanggeschoben werde, zu Gefäßverletzungen kommen und deshalb gegebenenfalls eine Notoperation in einem anderen Krankenhaus erforderlich werden könne. Dies bewerteten die Richter als ausreichend, da dem Patienten nicht jedes Risiko in allen denkbaren Erscheinungsformen dargestellt werden müsse, sondern es ausreichend sei, ihm nur eine allgemeine Vorstellung von dessen Art und Schwere des Eingriffs zu vermitteln. Danach müssten weder die gefährdeten Blutgefäße und die in Betracht kommenden Verletzungsarten medizinisch exakt bezeichnet noch alle denkbaren Folgen im Detail geschildert werden.

Weiterhin ging das Gericht davon aus, dass die - inhaltlich korrekte - Aufklärung auch von der Studentin im Praktischen Jahr durchgeführt werden durfte und dass auch die Anwesenheit eines Arztes ausnahmsweise entbehrlich war. Das OLG begründete seine Auffassung damit, dass die Aufklärung zwar grundsätzlich ärztliche Aufgabe sei, die zwar auf einen anderen Arzt, nicht aber auf nichtärztliche Hilfspersonen übertragen werden dürfe. Dies beruhe auf dem Gedanken, dass die Aufklärung medizinische Kenntnisse voraussetze, die bei nichtärztlichem Personal grundsätzlich nicht erwartet werden könnten. Die Aufklärung durch einen Medizinstudenten im Praktischen Jahr könne aber der ärztlichen Aufklärung bei Vorliegen bestimmter Voraussetzungen gleichstehen. Nach § 3 Abs. 4 Satz 2 der Approbationsordnung für Ärzte (ApprÖ̈) können und sollen Medizinstudenten im Praktischen Jahr entsprechend ihrem Ausbildungsstand unter Anleitung, Aufsicht und Verantwortung des ausbildenden Arztes innen zugewiesene ärztliche Verrichtungen durchführen. Dies entspreche auch dem Zweck des 
Praktischen Jahres, die Anwendungen der während des vorhergehenden Studiums erworbenen ärztlichen Kenntnisse zu erlernen und damit die praktischen Fähigkeiten und die klinische Erfahrung zu erwerben, die nach $\S 4$ Abs. 2 Satz 2 Bundesärzteordnung (BÄO) und Artikel 24 Abs. 3 Buchstabe d) der Richtlinie 2005/36/EG in der medizinischen Ausbildung vermittelt werden müssten. Diese Voraussetzungen erachtete das Gericht im vorliegenden Fall als gegeben: So hatte die Studentin den theoretischen Teil des Praktischen Jahres bereits absolviert und sich während der Famulatur mit Herzkatheteruntersuchungen befasst und diese bereits in Patientengesprächen erläutert. Ferner hatte sie den entsprechenden Aufklärungsbogen zuvor mit einem Arzt durchgearbeitet und besprochen, worauf besonders zu achten sei. Dem konkreten Aufklärungsgespräch waren zudem bereits mehrere Gespräche vorangegangen, bei denen die Studentin zugehört hatte bzw. von einem Arzt angeleitet worden war. Vor diesem Hintergrund erachtete es das Gericht auch als unerheblich, ob bei dem konkreten Aufklärungsgespräch ein Arzt anwesend war oder nicht, da es sich bei der Herzkatheteruntersuchung um einen Routineeingriff handele, über den die Studentin schon mehrfach ohne Beanstandungen aufgeklärt habe und bei dem es - anders als beim Eingriff selbst - nicht zu einem unvorhergesehenen Notfall kommen könne, der das sofortige Eingreifen eines Arztes erforderlich machen würde.

\section{Fazit}

Die Entscheidung des OLG Karlsruhe zeigt, dass es unter besonderen Umständen zulässig sein kann, die Patientenaufklärung auch auf einen Medizinstudenten zu übertragen, wenn sie seinem Ausbildungsstand entspricht und unter Anleitung, Aufsicht und Verantwortung des ausbildenden Arztes stattfindet. Die Entscheidung des OLG Karlsruhe steht damit auch im Einklang mit den Neuregelungen des Patientenrechtegesetzes, auch wenn sie sich nicht ausdrücklich damit auseinandersetzt. In der zum 26.02.2013 in Kraft getretenen Vorschrift des $\S 630$ e Abs. 2 des Bürgerlichen Gesetzbuches (BGB) heißt es ausdrücklich, dass die Aufklärung durch den behandelnden Arzt oder eine Person erfolgen muss, die über die zur Durchführung der Maßnahme erforderliche Ausbildung verfügt. Bis zuletzt hatte der Gesetzentwurf noch die Befähigung zur Durchführung der Maßnahme vorgesehen. Nach der Gesetzesbegründung soll jedoch die Aufklärung auch durch eine Person erfolgen können, die aufgrund ihrer fachlichen Ausbildung die notwendige theoretische Befähigung zur Durchführung der vorgesehenen Maßnahme erworben hat, auch wenn sie möglicherweise noch nicht das Maß an praktischer Erfahrung aufweist, das für die eigenständige Durchführung der Maßnahme selbst unverzichtbar ist. Durch die geforderte „Ausbildung“ ist nach Auffassung des Gesetzgebers sichergestellt, dass die Person über die nötigen Kenntnisse verfügt, um den Patienten umfassend über sämtliche für die Durchführung der Maßnahme wesentlichen Umstände aufzuklären. Mit dieser Kehrtwendung im Gesetzgebungsverfahren verlangt der Gesetzgeber damit keinen Facharztstandard bei der ärztlichen Aufklärung; vielmehr reicht es aus, wenn die aufklärende Person jedenfalls eine theoretische Befähigung zur Durchführung der vorhergesehenen Maßnahme erworben hat. Es ist daher nicht erforderlich, dass die aufklärende Person den betreffenden Eingriff selbst(-ständig) bereits durchgeführt hat, sondern dass sie die notwendigen Kenntnisse und theoretischen Grundlagen verinnerlicht hat. Dies trifft regelmäßig auf die in der Weiterbildung zum Facharzt stehenden Assistenzärzte zu, kann aber nach der vorliegenden Entscheidung des OLG Karlsruhe in geeigneten Ausnahmefällen auch für Studenten im Praktischen Jahr gelten.

Dennoch lässt sich aus dem Urteil nicht die Konsequenz ableiten, dass künftig standardmäßig die Aufklärung durch Medizinstudenten im Praktischen Jahr übernommen werden könnte. Letztlich entscheiden der verantwortliche Arzt im Einzelfall und die persönliche Qualifikation und der Kenntnisstand des jeweiligen Studenten. Den Studenten im Praktischen Jahr sollte daher regelmäßig die Gelegenheit gegeben werden, bei Aufklärungsgesprächen zunächst passiv mitzuwirken. Anschließend können sie unter Anwesenheit und Anleitung des ausbildenden Arztes Aufklärungsgespräche selbst führen. Mit zunehmender Sicherheit und Routine kann sich die Kontrolle durch den Arzt auf gelegentliche Stichproben beschränken, so dass eine ständige Anwesenheit des Arztes nicht erforderlich ist. Letztlich ist jedoch immer zu berücksichtigen, dass die Verantwortung für den Inhalt und Umfang des Aufklärungsgesprächs der Arzt trägt, so dass im Zweifel nach Aufklärung durch einen Medizinstudenten beim Patienten nachgefragt werden sollte, ob dieser die Aufklärung vollständig verstanden hat bzw. ob Fragen verblieben sind. Bei Vorliegen dieser Voraussetzungen stellt die Übertragung der Aufklärung auf Medizinstudenten im Praktischen Jahr eine grundsätzlich begrüßenswerte Schulung dar, die nicht nur zu einer Entlastung der Ärzte bei Personalmangel führen kann, sondern auch den Studenten die notwendigen praktischen Erfahrungen verschaffen kann. Dies gilt insbesondere vor dem Hintergrund, dass in der medizinischen Ausbildung bislang offenbar zu wenig Wert und Zeit auf die Vermittlung der im Aufklärungsgespräch erforderlichen Fähigkeiten gelegt wird. Dieser Aspekt sollte jedoch nicht vernachlässigt werden, da das Aufklärungsgespräch wichtiger Bestandteil der ärztlichen Behandlung ist und bei Aufklärungsfehlern unter Umständen erhebliche Schadensersatz- und Schmerzensgeldforderungen im Raum stehen können. Zudem stellen Rechtsprechung und Gesetzgeber inzwischen immer höhere Anforderungen an die Patientenaufklärung, so dass eine Verinnerlichung der Grundsätze bereits während des Studiums notwendig ist, um die erforderliche Sicherheit im Aufklärungsgespräch zu erwerben. Dies gilt seit Einführung des Patientenrechtegesetzes erst recht. 
Korrespondenzadresse:

Dr. iur. Albrecht Wienke

Wienke \& Becker - Köln, Bonner Straße 323, 50968 Köln Awienke@Kanzlei-WBK.de

\section{Bitte zitieren als}

Wienke A, Sailer R. Wirksame Aufklärung durch Medizinstudentin im Praktischen Jahr. GMS Mitt AWMF. 2014;11:Doc2.

DOI: 10.3205/awmf000291, URN: urn:nbn:de:0183-awmf0002916
Artikel online frei zugänglich unter

http://www.egms.de/en/journals/awmf/2014-11/awmf000291.shtml

Eingereicht: 25.03 .2014

Veröffentlicht: 26.03 .2014

\section{Copyright}

(C)2014 Wienke et al. Dieser Artikel ist ein Open Access-Artikel und steht unter den Creative Commons Lizenzbedingungen

(http://creativecommons.org/licenses/by-nc-nd/3.0/deed.de). Er darf vervielfältigt, verbreitet und öffentlich zugänglich gemacht werden, vorausgesetzt dass Autor und Quelle genannt werden. 